\title{
LA MÉDIATION, ENTRE IDÉAL THÉORIQUE ET APPLICATION PRATIQUE
}

\section{Paul Rasse ${ }^{1}$}

Comme toute notion à la mode, le terme de "médiation culturelle" devient difficile à cerner. Sa définition oscille entre deux extrêmes : d'un côté, une approche théorique très générale mêlant les sciences sociales à la philosophie, de l'autre, des descriptions, des comptes rendus d'expérience très pragmatiques, centrées sur les réalisations novatrices du moment, et parfois leur analyse. Le risque est que le lien ne se fasse pas, entre le ciel évanescent des idées et le monde concret des praticiens, abandonnés à eux-mêmes, dont la seule quête est celle de l'efficacité, pour laquelle ils mobilisent un appareillage toujours plus sophistiqué en perdant de vue les enjeux et la finalité profonde de leur travail.

Après avoir cherché à définir chacun de ces deux pôles, nous nous efforcerons de tenir les deux bouts, de ne pas renoncer à l'expérience, mais de donner du sens aux pratiques, en jouant du débat théorique et des utopies auxquelles il renvoie, en référant à l'histoire,

\footnotetext{
1 Professeur au Département Art, Communication, Langages de l'Université de Nice Sophia Antipolis.
}

Recherches en communication, $\mathrm{n}^{\circ} 13,(2000)$. 
celle des animateurs socioculturels, ou plus ancienne encore de l'espace public, en n'abandonnant jamais la discussion sur les enjeux que recouvrent la légitimation et le partage des cultures.

\section{Utopie de la médiation}

La définition du Dictionnaire de la communication relève du pôle théorique, là tout est médiation, à commencer par le langage et le symbolique "car ils assurent au cours de l'usage qu'en est fait par les sujets, l'appropriation singulière des codes collectifs". S'y ajoutent les formes de sociabilité, les structures de la communication interpersonnelle ou médiatiques qui relient les personnes entre elles et au collectif. L'individu doit refouler cette part irréductible que l'on nomme le réel pour accéder au symbolique qui assure son intégration et tient toute la communauté. "La médiation, dialectique, entre le singulier et le collectif, suppose ainsi l'oubli du singulier pour la mise en œuvre d'une forme collective de l'appartenance"'. En même temps, comme le montre bien Habermas, la conception moderne des rapports entre l'art et son public, repose sur l'émergence du sujet, de l'individu. Il se découvre une sensibilité personnelle et fait l'expérience de la subjectivité d'abord en privé, avant d'en débattre dans l'espace public où se forge l'opinion commune à l'égard des réalisations culturelles ${ }^{2}$. Aussi la médiation aurait, elle, pour fonction d'assumer cette tension entre l'individuel et le collectif.

Jean Caune élargit la médiation à l'histoire individuelle et collective, qui relie les hommes aux générations passées, à travers l'héritage, le patrimoine, et leur permet de se projeter dans l'avenir. Elle est considérée comme "mise en rapport entre ce qui a été et ce qui devient". La médiation culturelle comme expérience esthétique sensible, serait alors tendue entre quatre pôles opposés deux à deux selon deux grands axes : en abscisse "la relation des sujets à travers la relation interpersonnelle", en ordonnée, "la relation transcendante des membres d'une collectivité à leur passé et à leur devenir"3.

1 Dictionnaire encyclopédique des sciences de l'information et de la communication, sous la direction de B. LAMIzET et A. SILEM, Paris, Ellipses, 1997, p. 364.

2 J. Habermas, L'espace public, Paris, Éd. Payot, 1996, (lère édition en allemand 1962, en français 1978), p. 55.

3 J. CAUne, Pour une éthique de la médiation, le sens des pratiques culturelles, Grenoble, PUG, 1999, p. de couverture, p. 12 ou encore p. 194. 
Finalement, la médiation ainsi définie dans son acception la plus large, recouvre l'idée de culture au sens anthropologique du terme. Elle est ce qui relie les hommes entre eux, pour donner du sens à leur existence personnelle, et à l'humanité qu'ils constituent ensemble depuis la nuit des temps. Si l'on définit la culture "comme cet ensemble complexe englobant les savoirs, les croyances, les arts, la morale, les lois, les coutumes ainsi que les autres capacités et habitudes acquises par l'homme en tant que membre d'une société"'; alors, la médiation est le liant sensible et symbolique. Elle assure la cohésion sociale par l'adhésion de tous à chacun des éléments constitutifs de la culture commune. Elle fait que fonctionne cette définition qu'en donne Braudel : "une même façon de naître, de vivre, d'aimer, de se marier, de penser, de croire, de rire, de se nourrir, de se vêtir, de bâtir ses maisons et grouper ses champs, de se comporter les uns visà-vis des autres"2.

Le risque est de se perdre dans un espace marécageux, improbable, aux frontières indéfinies où se mêlent la terre et les eaux qui s'y infiltrent. Si bien que l'on peut voir et étudier partout des formes de médiation, mais ne jamais réussir à s'en saisir, à la drainer et à la canaliser pour la rendre plus vive et pouvoir irriguer des espaces desséchés. Car l'idée de médiation recouvre une forme d'intentionnalité. On prend conscience de son importance, si elle disparaît, comme on ne prend réellement conscience de l'importance de l'eau dans le sol qu'à partir du moment où les plantes flétrissent et où se posent des problèmes d'arrosage. La médiation devient perceptible, en négatif, à partir du moment où elle fait défaut, où quelque chose manque, et où la société cherche des remèdes.

Si elle plane dans le ciel des idées, la médiation devient alors une essence supérieure, immanente, transcendante, propice à toutes les utopies de la communication qu'énoncent Philippe Breton ${ }^{3}$ ou Victor Scardigli ${ }^{4}$. Elle est une panacée. Elle intervient dans le jeu symétrique où s'opposent espérances et craintes que suscite le mouvement de la société. Elle apporte la liberté, l'autonomie, l'intégration face aux

1 C. TAYLOR, The Origin of Culture, cité in "La rencontre des cultures", Sciences Humaines, $\mathrm{n}^{\circ} 16,1992$, p. 30.

2 F. Braudel, L'identité de la France, Espace et Histoire, Paris, Éd. Champs Flammarion, 1990, p. 81.

$3 \mathrm{Ph}$. BRETon, Utopie de la communication, Paris, La Découverte, 1993.

4 V. SCARDigli, Le sens de la technique, Paris, PUF, 1992. Voir notamment les chapitres sur les 7 miracles p. 53 et sur les 7 plaies p. 72 . 
angoisses de la ségrégation, de l'exclusion et de la relégation qui plane sur chacun. À la solitude et à l'isolement, elle oppose la convivialité, la rencontre, l'échange. Elle défend les exigences du savoir et la maîtrise des connaissances contre l'inculture, l'abêtissement et l'engloutissement dont nous menace l'inflation mass médiatique. Elle vise à l'égalité et à la promotion de tous contre la destruction et la domination d'une intelligentsia. Elle est du côté de la prospérité, de la jouissance partagée, la délectation, contre l'appauvrissement et la dégénérescence. Et l'on pourrait ainsi ne jamais finir d'allonger la liste des valeurs que défend la médiation. Si cette conception a l'avantage de toutes les utopies, "donner du sens aux projets des hommes"1, c'est à condition de ne pas être coupée du monde vécu, notamment, pour ne pas abandonner les acteurs sur le terrain aux seuls impératifs de l'action et de la quête de résultats tangibles.

\section{La médiation orthopédique}

A l'autre bout, dans le champs des comptes rendus pragmatiques, la médiation culturelle se définit par des pratiques concrètes, des techniques et des savoir-faire mis en œuvre par des professionnels. Cette fois, elle repose sur l'idée d'intervention sociale dans une perspective non plus utopique et idyllique, mais orthopédique et efficace. Il s'agit, en effet, de réduire des fractures tangibles, là où elles se produisent, de raccommoder le tissu social dans les quartiers en grandes difficultés, d'amener les communautés ethniques à se supporter, de faciliter l'acculturation des migrants, de tisser des liens entre la cité et les citoyens, de cultiver la science, de donner le goût de la culture légitime.

Dans le domaine des savoirs savants, elle doit rattraper ce que l'école a raté. Il faut réorganiser les connaissances mal digérées, rectifier les erreurs de compréhension, chasser les fausses croyances, faire la guerre à l'irrationnel, expliquer, traduire les savoirs incompréhensibles d'expert pour les rendre accessibles au plus grand nombre, susciter la curiosité, l'envie d'apprendre, et enfin provoquer une attitude positive, ouverte, à l'égard de la science et des techniques.

1 P. Delcambre, "Penser les pratiques culturelles en se saisissant du concept de médiation”, Études de communication, n²1, 1998, p. 137. Voir aussi Ph. BRETON, Utopie de la communication, op. cit., p. 149. 
Dans le domaine des arts, la médiation se bat contre les penchants de la plèbe à préférer les jeux du cirque, à se laisser enchanter par les attractions scintillantes des parcs de loisirs ou des soirées de télévision. Il faut solliciter les visiteurs, fidéliser les spectateurs, traquer les non-publics, cibler la communication, éduquer, rééduquer, développer le désir et le plaisir des belles choses, amener le peuple à comprendre et à respecter la création contemporaine, à défaut de l'aimer, alors qu'il est enclin à s'en détourner et à l'ignorer, parfois à s'en moquer, jusqu'à la haïr.

Cette fois, la médiation mobilise des technologies bien concrètes ; après l'audiovisuel et la vidéo, l'informatique et la PAO, vient le temps du multimédia, des CD Rom et maintenant de l'Internet. La mise en scène, l'exposition, les conférences, le spectacle, la fête, les conjuguent entre elles selon une alchimie savante et sans cesse renouvelée. Il n'est plus question que d'accrochages, de tournage de séquence vidéo, d'écriture multimédia, d'arborescence logistique, de circulation du public, de stratégies de communication, de publicité, de marketing mixte. On est loin, bien loin des utopies précédentes; cette fois, si les nouvelles technologies de la communication font rêver, c'est tout simplement pour leur efficacité supposée. Elles laissent seulement espérer qu'elles résoudront des problèmes pratiques, là où les autres ont déjà échoué. La séduction, le jeu, le défi, occupent une place centrale qui n'est pas sans rappeler les artifices orthopédiques des orthophonistes, des ergothérapeutes psychiatriques, des kinésithérapeutes pour rééduquer presque à leur insu les patients, enfants et parfois adultes aussi. Les techniques d'évaluation sont censées, s'efforcent de mesurer les résultats, de déceler les mauvaises séquences de médiation, et de mettre en évidence les bonnes, d'en expérimenter de nouvelles ${ }^{1}$, jusqu'à une véritable taylorisation des processus, que l'on décompose en éléments isolés, dont on évalue scientifiquement les performances avant de généraliser les meilleures solutions.

Là, les résultats sont tangibles, quantifiables, même si au plan qualitatif, ils sont souvent bien maigres, comme le montrent par exemple les statistiques du ministère de la Culture, quand elles indiquent qu'en trente ans les structures du public de la culture

1 A. Giordan, P. RASSE, Culture, communication, évaluation, Nice, Z'Editions., 1987. E. CAILLET, A l'approche du musée, la médiation culturelle, Lyon, Éd. PUL, 1996, p. 163. 
légitime n'ont quasiment pas changél. Et l'on peut en fin de compte s'interroger sur l'intérêt des dispositifs pratiques de cette médiationlà, qui se perd en conjectures et en gesticulations destinées pour le moins à rassurer les politiques quant au bon usage des finances publiques.

\section{L'orgueil des animateurs}

Nous avons défini la médiation à partir de ses deux extrêmes, au risque de forcer le trait pour les rendre perceptibles et interpréter les pratiques et les discours qui se placent sous sa coupe. Maintenant, pour donner du relief à l'ensemble et le ressaisir dans son épaisseur, nous nous intéresserons aux ancêtres, les animateurs socioculturels, moins pour leur rendre hommage que pour tirer quelques enseignements de leur disparition de la scène publique. Figure marquante des années soixante-dix, ils ont connu leur heure de gloire au meilleur du "well-fare of state". La réintégration des exclus de la croissance est déjà une idée prégnante de l'époque ${ }^{2}$. Il s'agit de mieux répartir les fruits des trente glorieuses, mais aussi d'étendre le bonheur des classes moyennes au partage des biens culturels, après l'accession à la consommation de biens matériels. Malraux a prévu pour cela des équipements prestigieux, les fameuses maisons de la culture. Lieux de création et de diffusion artistique, implantés dans les grandes agglomérations du pays, elles se déclinent en équipements de plus en plus sommaires au fur et à mesure que l'on s'éloigne du centre. Les Maisons des jeunes et de la culture, implantées au cœur des villes de moyenne importance ou dans des quartiers périphériques mais anciens et structurés, sont encore assez bien équipées. Les banlieues les plus déshéritées n'ont elles droit qu'à des préfabriqués banalisés comme les fameux "1000 clubs" construits en série à mille exemplaires et

1 Les disparités sociales n'ont pas diminué ; “on peut même penser", écrivait Olivier Donnat en 1993 à propos des musées, "si l'on retient comme indicateurs l'évolution des écarts entre les taux de pénétration chez les ouvriers et les employés d'une part et chez les cadres supérieurs d'autre part, qu'elles ont tendance à s'accentuer". O. Donnat, "Les Publics des musées en France", Publics et musées, $\mathrm{n}^{\circ} 3$, PUL, 1993, p. 35. Et les statistiques publiées depuis par O. Donnat ne signalent pas non plus de changements significatifs. Cf. O. Donnat, "Les pratiques culturelles des français, Enquête 1997", La documentation française, 1998.

2 Voir notamment sur le sujet: C. PATRIAT, La culture un besoin d'État, Paris, Hachette, 1998. 
installés en hâte sur des terrains vagues, sans équipement ni personnel qualifié. Là, il n'est plus question de diffusion, encore moins de créations, juste d'offrir aux jeunes et aux femmes désœuvrés des lieux de rencontre suscités par des activités occupationnelles.

Pour l'ensemble, et sans doute dans l'esprit de cette époque fortement marquée par mai 68 , le projet est d'abord celui d'un manifeste d'intervention sociale. Il s'agit d'inventer une nouvelle culture qui intégrerait la culture ou au moins les aspirations de ce que l'on appelle déjà les "non publics", parce qu'ils ne fréquentent pas les lieux artistiques légitimes. "Cette démarche, résume Claude Patriat, abouti à remettre l'intervention culturelle sur ses pieds, en donnant la priorité à la population, dont il s'agit de découvrir et de susciter les attentes... Cette stratégie privilégiant l'animateur par rapport au créateur..."l.

Jusqu'alors, les mouvements d'éducation populaire issus du mouvement ouvrier, dans le sillage du front populaire (la semaine de 40 heures et les premiers congés payés) et de la libération (création des comités d'entreprise) s'étaient battus pour que le peuple accède à la culture cultivée. Elle est synonyme pour eux de promotion sociale au même titre que l'a été, l'instruction publique. Elle passe par l'accession des travailleurs à des formes de savoirs et de culture dont ils étaient jusque-là exclus et qu'ils ne remettent pas en question.

Les animateurs socioculturels sont en rupture avec cette conception. Althusser vient de publier son fameux article sur les appareils idéologiques d'État. Il y défend que l'école et les institutions culturelles sont comme l'Église autrefois, au service de la domination idéologique d'une minorité. La culture artistique officielle, celle des beaux-arts, est entachée par ses origines aristocratiques et bourgeoises ; les artistes ne sont plus conviés que s'ils sont en rupture avec leur position sociale et leur statut passé. A la création, on préfère d'ailleurs la créativité, chacun possède un potentiel qu'il convient de cultiver et d'exprimer plutôt que de l'abandonner à des artistes. Ils défendent une conception de la culture prosaique inscrite dans la cité et dans la vie des hommes qui l'habitent. Ce que Francis Jeanson appelle la "culture vivante" qu'il définit ainsi : "ce n'est ni les "humanités" ni les "beaux-arts", ce n'est plus celle de "l'honnête homme" au sens classique du terme, qui pouvait naguère s'imaginer de plain-pied avec la production intellectuelle et artistique de son

${ }^{1}$ C. Patriat, op. cit., p. 119. 
époque, ce n'est pas davantage celle du savant contemporain, de plus en plus contraint de s'enfermer dans telle branche de telle sousspécialité s'il veut conserver sa valeur marchande au regard du système. La culture vivante, nous ne pouvons désormais la concevoir qu'en termes de rapports humains et de vie quotidienne au sein de nos cités" ". Et de le confirmer dans un manifeste pour la formation des animateurs : "le culturel, c'est de moins en moins la rituelle diffusion -consommation de produits finis relevant des beaux-arts... c'est de plus en plus la création commune et quotidienne d'une socialité qui apparaît comme indispensable chaire de tout engagement politique défini : sa condition de liberté en même temps que d'efficacité "2.

L'institution muséale elle-même est vivement critiquée comme temple célébrant la culture des élites. Faut-il comme Erostate, se demande Clair, incendier le sanctuaire ? Et bon nombre d'artistes, d'intellectuels, de développer des arguments selon lesquels "il ne peut y avoir de progrès dans les arts ou la démocratisation des arts tant que le Louvre n'aura pas été brûlé"3. En tout cas, déclare Hugues de Varine au Congrès de l'ICOM à Grenoble en 1971, "le musée consacré à la tradition est en train de mourir...". Et de conclure : "La révolution du musée sera radicale ou le musée disparaîtra" 4 . Les conservateurs les plus engagés inventent un nouvel idéal de musée, l'écomusée dont les animateurs sont au service d'une population. Ils sont chargés par la communauté de recenser, de conserver et de révéler les éléments de sa culture pour en dégager les caractéristiques populaires, rurales, artisanales ou industrielles ${ }^{5}$.

"C'est à l'institution elle-même, proclamait Duncan Cameron, de financer la révolution, en donnant aux artistes et aux critiques sociaux des occasions d'être entendus, d'être exposés et de contester les valeurs reconnues et les institutions en place". Dans les centres culturels, le point de vue est le même ; l'animateur est un agitateur social, défend Jean Hurstel, "un organisateur de fêtes, de manifestations collectives fruits de la concertation et du travail d'une communauté (carnaval, feux de la Saint-Jean)... C'est avant tout le militant

1 F. JEANSON, L'action culturelle dans la cité, Paris, Éd. du Seuil, 1973, p. 54.

2 Ibid., p. 210.

3 D. CAMERon, Le Musée Temple ou Forum, 1971, cité in Vagues, "Une anthologie de la nouvelle muséologie, Ed. W / MNES, 1992, p. 89.

4 Rapporté par H. DE VARINE, in Vagues, "Une anthologie de la nouvelle muséologie, op. cit., p. 448.

5 H. DE VARINe, "Le musée au service de l'homme et du développement", Museum, vol. XXV, $n^{\circ} 4,1973$. 
d'un changement social"'. Les lieux d'animation se veulent d'abord des lieux d'agitation sociale auxquels participent éventuellement les artistes les moins conventionnels, ce qui finit par inquiéter les élus. Le tonitruant maire de Nice, Jacques Médecin, est l'un des premiers à monter au créneau, il veut fermer la maison des jeunes de Gorbella, l'accusant d'être un foyer insurrectionnel gauchiste. À Chalon, Jeanson qui dirige le projet de création d'une maison de la culture est lui aussi violemment attaqué par le maire de la ville qui veut sa démission. Ailleurs, la répression sera plus subtile et non moins efficace. Les élus ont bien compris les enjeux politiques et le risque d'abandonner la médiation à des structures indépendantes, bien que directement financées par l'État et les collectivités locales. Dès lors, ils n'auront de cesse d'en reprendre le contrôle. Ils y parviendront en municipalisant les structures et en fonctionnarisant les animateurs.

L'échec de l'animation ne tient pas non plus qu'à cela ; il faut aussi prendre en considération le contexte de crise économique. Les années 80 se veulent pragmatiques, là où tous voyaient auparavant les risques et le prix à payer pour toute programmation exigeante, alliant création artistique et expérimentation sociale, tous ne voient plus maintenant que des coûts prohibitifs pour des salles trop vides, une fréquentation trop élitaire, des déficits à éponger et de la mauvaise gestion. Les moyens sont en baisse, les animateurs sont maintenant sommés de faire du chiffre et la preuve de l'utilité sociale des équipements. Il faut remplir les établissements, trouver et appliquer des recettes qui marchent, répondre à la demande des associations et des populations locales, promouvoir les pratiques amateurs, les activités centrées sur le corps et le bien-être personnel (l'expression corporelle, le yoga, la gymnastique, la danse...) organiser des cours de couture ou de poterie, des stages de tissage ou d'expression plastique. La médiation artistique qui n'était déjà plus la priorité de ces institutions est abandonnée. Les maisons de la culture se centrent sur la programmation de spectacles et d'expositions, sans plus avoir ni politique de diffusion, ni ambition quant aux fameux non-public. De leur côté, les MJC et autres maisons de quartier se centrent sur des activités socio-éducatives de médiation entre les individus et les communautés, mais renoncent aux artistes et à la médiation de leurs créations.

1 Texte publié en 1975, cité par C. PATRIAT, op. cit., p. 127. 
Les animateurs rêvaient d'une nouvelle culture, de bouleverser les règles de l'esthétique quitte à se passer des artistes, et commis par là sans doute un péché d'orgueil, d'avoir voulu prendre la place des dieux, de ceux dont ils avaient la mission de médiatiser les créations. Jack Lang aura beau jeu de le leur reprocher, et pour marquer le coup, d'imposer des artistes à la tête de tous les grands établissements, à commencer par les maisons de la culturel.

Est-ce pour que tout cela ne puisse plus jamais se reproduire, que le terme de médiation, (étymologiquement centré sur la fonction qu'il rend incontournable) va progressivement s'imposer et se substituer à celui d'animation? En tout cas, le succès et l'essor du projet de médiation viendront moins du domaine des arts, que de celui des sciences, dans le sillage du mouvement de "mise en culture des sciences et des techniques"2. Il se développe à partir des années 80 , pour renouer les liens avec la recherche scientifique de plus en plus spécialisée, incompréhensible pour le profane, et en même temps, omniprésente dans son existence par les applications techniques qui bouleversent les modes de vie. En France, il est décidé de mettre en place sur tout le territoire des structures de médiation, de façon à contrebalancer la gigantesque Cité des sciences et de l'industrie de La Villette. Il est notamment projeté de créer des centres de culture scientifique et technique (CCST) dans les grandes villes de province. Là, les maisons de la culture servent de modèle. Des préfigurations sont lancées un peu partout, rejointes par les musées des sciences tombés en désuétude; (ils y voient l'occasion de retrouver une fonction sociale et les moyens financiers de leur rénovation). Cette fois, il ne peut être question de se passer des principaux acteurs intervenant dans le champ social à médiatiser, c'est-à-dire des scientifiques qui produisent les savoirs.

D'innombrables expériences seront menées un peu partout. Chaque fois la réflexion s'engage sur les finalités, les objectifs et les moyens à mettre en œuvre. Peu à peu, l'idée de médiation s'étoffe et la figure professionnelle de médiateur prend corps, se substituant à celle d'animateur scientifique. Elle s'étend peu à peu aux musées de société, techniques et industriels, au patrimoine, puis gagne tous les domaines de la culture, jusqu'au succès qu'on lui connaît.

1 Entretien avec Jack Lang, Mei, Média et information, $n^{\circ} 3$, Éd. Université de Paris VIII, 1995.

2 Selon l'expression de Jean Marc Lévy-Leblond, Mettre la science en culture, Nice, Éd. Anaïs, 1986. 


\section{Le projet de médiation esquisse une définition}

Entre une conception de la médiation trop théorique, trop générale, trop utopique pour être utile et une approche pragmatique, trop pratique, engluée dans le quotidien, jusqu'à perdre le sens de ses activités ; après l'expérience des animateurs disparus dans la récession et la confusion, qui voulaient réinventer la culture et se passer des artistes, quelle définition peut-on encore tenter ?

La médiation n'est pas un concept, une théorie organisée, relativement solidifiée et prête à l'emploi pour analyser l'espace social ; elle recouvre au contraire un champ de débat théorique et de pratiques professionnelles sur les relations entre la culture et son public. Elle est cet espace de communication où se tissent les liens des visiteurs avec les musées et le patrimoine, des spectateurs avec le théâtre, des auditeurs avec la musique, bref, des publics avec les formes d'expression artistique légitimes, auxquelles on peut encore ajouter celles qui prétendent à cette légitimité : les cultures populaires, le design industriel, la science... Le jeu est ouvert, en filigrane, on peut lire la dynamique culturelle d'une société. Pour donner du corps à cette proposition, nous nous contenterons de pointer ici et là quelques-uns des éléments du débat, et de répertorier quelques-unes des pratiques qui s'y rattachent.

Le débat sur la médiation ne peut faire l'économie d'un débat sur les deux champs qu'elle est censée mettre en relation, celui de la culture et celui du public. Il convient de considérer comment est-ce qu'une société produit sa culture artistique, mais aussi scientifique ou populaire... Comment est-ce qu'elle la finance et dans quelle perspective est-ce qu'elle le fait. La médiation ne peut pas non plus faire l'impasse sur l'esthétique, non seulement sur le jugement de goût, mais sur les conditions de sélection, de promotion, de légitimation du travail de tel ou tel artiste, et plus généralement de toutes les productions culturelles. La médiation est aussi cet espace incertain par lequel remontent les attentes et les goûts du public, jusqu'à influencer la création. On pense bien sûr aux études de public, aux évaluations, mais aussi aux contacts que provoquent les situations de médiation. Reste ensuite à savoir s'il faut en tenir compte dans la programmation. Évidemment, l'esthétique ne peut plus être l'apanage d'une petite élite qui s'est approprié le privilège des princes et veut le garder pour elle seule. Pour autant, il faut se méfier du "grand public", cette notion mass médiatique qui impose de s'aligner sur le plus petit 
dénominateur commun d'exigence, et de "sucrer" les productions toujours davantage, de façon à les rendre plaisantes au goût de tous ? Pour toutes ces questions, il n'est pas de réponse universelle, seulement des débats à avoir, des compromis à construire, des expériences à mener...

Les médiateurs ont souvent tendance à considérer les arts ou encore la science et les autres manifestations de la culture, comme des objets réifiés, comme une boîte noire, sans s'interroger sur les conditions de leur production; si bien qu'il suffirait de mobiliser les techniques adéquates, les savoir-faire pertinents dans une bonne campagne de communication, pour les faire comprendre et apprécier sans jamais se poser la question sur leur statut. L'idée de médiation que nous défendons est beaucoup plus vaste. Elle intègre une mise en question du statut de l'art et de la culture, elle s'efforce de retrouver le point de vue global des animateurs centré sur le public saisi dans son contexte social, mais d'éviter les dérives qui les ont conduits à se passer des artistes. Cette fois, la création et le patrimoine artistique sont au centre, le sujet même de la médiation.

Denis Guedj et Elisabeth Caillet défendent l'idée selon laquelle le médiateur est un "passeur", Il se différencierait de l'enseignant en ayant à sa disposition un arsenal technologique sophistiqué, mais surtout en n'ayant pas d'obligation de contenu, si bien que contrairement à l'école contrainte par des programmes de connaissances, il pourrait "travailler pour que transparaisse le plaisir, le désir". Cette vision est par trop restrictive, le réduit à un super technicien/conférencier/conteur dont l'action se situe exclusivement à l'interface entre du public et des cuvres ou de la science ${ }^{1}$. La médiation ne peut se limiter à cela, elle recouvre l'ensemble des dispositifs de communication des organisations culturelles saisies dans leur complexité.

Alors que dans l'entreprise, on sait maintenant que communication interne et communication externe sont étroitement liées, les institutions culturelles continuent de distinguer la médiation pédagogique interne, des relations publiques chargées des contacts avec la presse et la publicité. Et pourtant, le succès de la médiation dépend de la coordination et de la redondance de l'ensemble des processus de communication, qui doivent en outre engager toute l'institution dans une réflexion sur ses missions vis-à-vis du public.

1 E. CAILLET, op. cit., p. 21. D. GuEDJ, Lettre des musées de France, DMF,1991. 
La programmation et la création ne relèvent évidemment pas de la médiation, mais cette dernière a néanmoins pour mission de cultiver le goût du public chez les programmateurs ou chez les artistes, en se faisant l'écho de ses attentes et de ses demandes, en les informant des études de publics, et plus généralement de l'ensemble des connaissances glanées ici et là sur celui-ci. Plus globalement encore, et pour autant que la médiation réussisse à engager toute l'institution dans une réflexion sur les mutations de la culture dans la société, elle lui offre l'opportunité d'évoluer, d'anticiper, d'innover, d'être au premier plan de la scène médiatique et de contribuer à ces mutations plutôt que de les suivre.

Il faut noter que la médiation est maintenant un champ de recherche officiel et en plein essor. Il se situe résolument au sein de la discipline universitaire des sciences de l'information et de la communication, mais fédère aussi des chercheurs en esthétique, en histoire de l'art et en sociologie du public. Il a ses groupes de travail (dont l'un au sein de la SFIC qui anime la recherche dans le champ SIC). Ses congrès, ses laboratoires, ses colloques où se débattent les problématiques de la médiation, où vivent, se réactivent, se confrontent et se nourrissent les débats. Mais la médiation, c'est aussi des métiers, des pratiques professionnelles, des techniques et des savoir-faire. Elle est cet espace d'usage des technologies de la communication destiné à provoquer, à porter ou à soutenir les rapports aux productions culturelles, l'exposition, la conférence, la visite guidée, les dispositifs multimédias, la vidéo, l'édition, la PAO. Et si le médiateur n'est généralement pas un spécialiste de leur usage, il dirige les réalisations qui les emploient en fonction d'un projet de médiation qu'il doit élaborer et partager avec les techniciens, de façon à donner du sens à chaque élément, comme à l'ensemble du dispositif.

Signe des temps, la médiation est maintenant enseignée à l'université dans la perspective de professionnalisation des étudiants. Les premiers niveaux, les plus anciens, forment des techniciens opérationnels (IUT) souvent encore appelés animateurs. Les formations les plus recherchées se situent maintenant à bac +4 (maîtrise professionnalisée, MST ou IUP), mais surtout à bac +5 dans une bonne quinzaine de Masters (DESS et DEA, notamment en muséologie) qui forment des cadres. 


\section{Conclusion : enjeux de la médiation}

Claude Patriat regrette que le médiateur ne soit plus un militant du changement social comme le défendait Hurstel à propos des animateurs'. Mais n'est-ce pas avoir une vision trop messianique, et de l'histoire, et de cette profession, censée révéler au peuple son oppression, le conduire à une libération culturelle par laquelle il se réapproprierait son expérience personnelle. Si le médiateur peut avoir un rôle de transformation sociale, c'est au sens où l'entendent Kant à propos des Lumières, ou plus tard Habermas avec le concept d'espace public ${ }^{2}$. Là, ce ne sont plus des tribuns qui engagent la foule dans une révolution, mais un long et imperceptible travail du corps social sur lui-même, parce qu'il invente des lieux publics, les salons, les cafés, les salles de théâtre et de concert, où il se découvre petit à petit en capacité de mettre en question la culture des élites, et où il fait l'apprentissage du sens critique, jusqu'à mettre en débat et en question la culture de son époque ${ }^{3}$.

La médiation serait alors cet espace où le public se fixe l'exigence de participer à la culture, parfois comme ignorant confronté à des sujets qu'il ne connaît pas, mais toujours en mesure de les discuter à partir de ce qu'il est lui-même. La vulgarisation traditionnelle considère que la personne est ignorante au sens de vide et qu'elle désire combler ce vide. Les approches didactiques adoptent parfois un principe plus complexe, selon lequel la personne serait encombrée d'un fatras de connaissances mal digérées, qu'il convient de rectifier et de compléter. La médiation, au sens où nous l'entendons, considère d'abord que la personne est différente, étrangère au lieu ou au sujet. Elle s'efforce de l'accueillir, d'expliquer, de traduire, dans un processus de négociation à partir de ce que chacun est, de ses capacités cognitives, de sa propre culture, de ses aspirations, de ses angoisses, de sa propre histoire, de son statut social. La médiation consiste alors à créer des espaces où le public se sente respecté et reconnu dans sa différence, d'abord pour l'attention dont il est l'objet,

1 J. HURSTEL, "Formation de l'animateur, $A T A C, \mathrm{n}^{\circ} 64$, janvier 1975, p. 27, cité par C. PATRIAT, op. cit., p. 127.

2 E. KANT, Réponse à la question : qu'est-ce que les lumières?, Éd. de La Pléiade, p. 211.

3 Voir : P. RAsse, Le musée à la lumière de l'espace public, Paris, L'Harmattan, 1999. 
pour cet effort d'hospitalité de l'institution qui l'accueille, qui tente d'expliquer, d'informer, de traduire.

Bien sûr, tout cela est encore à l'état d'esquisse. Il reste à inventer des espaces où le public puisse réellement construire sa propre opinion à partir des informations auxquelles il a accès grâce à l'effort de médiation et à partir de ce qu'il est, lui et la communauté des hommes à laquelle il appartient. Cela suppose de créer des espaces de confrontation entre lui et l'œuvre, lui et le sujet de l'institution culturelle, lui et les autres visiteurs, bref, à faire que les lieux de culture soient aussi des espaces publics. 